
Archive

University of Zurich

University Library

Strickhofstrasse 39

CH-8057 Zurich

www.zora.uzh.ch

Year: 2011

\title{
Bisshebung mit Komposit im Erosionsgebiss
}

Tauböck, T T ; Schmidlin, P R ; Attin, T

DOI: https://doi.org/10.1007/s11838-011-0121-8

Posted at the Zurich Open Repository and Archive, University of Zurich

ZORA URL: https://doi.org/10.5167/uzh-50266

Journal Article

Accepted Version

Originally published at:

Tauböck, T T; Schmidlin, P R; Attin, T (2011). Bisshebung mit Komposit im Erosionsgebiss. Wissen Kompakt, 5(3):23-32.

DOI: https://doi.org/10.1007/s11838-011-0121-8 


\section{Bisshebung mit Komposit im Erosionsgebiss}

T. T. Tauböck, P. R. Schmidlin, T. Attin

Klinik für Präventivzahnmedizin, Parodontologie und Kariologie,

Zentrum für Zahnmedizin der Universität Zürich

Korrespondenzadresse:

Dr. Tobias T. Tauböck

Klinik für Präventivzahnmedizin, Parodontologie und Kariologie,

Zentrum für Zahnmedizin der Universität Zürich

Plattenstrasse 11

CH-8032 Zürich

Schweiz

Tel: +41446343448

Fax: +41446344308

E-Mail: tobias.tauboeck@zzm.uzh.ch 
Dr. Tobias T. Tauböck, Jahrgang 1982, studierte von 2002 - 2007 Zahnmedizin an der Julius-Maximilians-Universität Würzburg und promovierte dort 2008 in der Abteilung für Funktionswerkstoffe der Medizin und der Zahnheilkunde. Seit Januar 2008 ist Herr Dr. Tauböck als Assistent in der Klinik für Präventivzahnmedizin, Parodontologie und Kariologie der Universität Zürich tätig. Seine Hauptarbeitsgebiete sind die präventive, restaurative und ästhetische Zahnheilkunde. Dr. Tauböck gewann 2010 den Forschungswettbewerb VOCO Dental Challenge als bester Nachwuchswissenschaftler. 


\section{Zusammenfassung}

Ein umfangreicher Bisshöhenverlust infolge fortgeschrittener Abrasion/Erosion stellt restaurativ eine schwierige Situation dar. Üblicherweise erfolgt die Wiederherstellung der ursprünglichen Bisslage mit indirekt hergestellten Werkstücken, die eine Präparation der Zähne erfordern und für den Patienten hohe Kosten verursachen. Der vorliegende Beitrag beschreibt eine vergleichsweise kostengünstige, noninvasive Alternative mit adhäsiven okklusalen Kompositrestaurationen zum direkten Aufbau der verloren gegangenen Zahnhartsubstanz. Um ein möglichst einfaches, effizientes und kontrolliertes Vorgehen zu gewährleisten, wird dabei eine auf einem individuellen Wax-up basierende Tiefziehschiene als Formhilfe verwendet.

\section{Schlüsselwörter}

Bisshebung, Komposit, Schienentechnik, Abrasion, Erosion 
In der täglichen Praxis werden vermehrt pathologische Zahnhartsubstanzdefekte beobachtet, die nicht auf Karies beruhen, sondern durch chemische (Erosion) und/oder mechanische (Abrasion, Attrition) Prozesse hervorgerufen werden. Eine Absenkung des Bisses infolge fortgeschrittener Zahnhartsubstanzverluste kann zu funktionellen und ästhetischen Problemen führen, die eine restaurative Intervention erfordern. Im folgenden Beitrag wird eine alternative Methode zur konventionellen Therapie mit Werkstücken beschrieben, bei der mit einer speziellen Schienentechnik und direkten Kompositaufbauten die verloren gegangenen Zahnanteile ohne Opferung von gesunder Zahnhartsubstanz ersetzt werden.

\section{Ursachenabklärung}

Nichtkariöse Zahnhartsubstanzdefekte werden durch Kontakt mit exogenen oder endogenen Säuren ohne bakterielle Beteiligung hervorgerufen (Erosion) oder sind primär durch mechanische Einflüsse bedingt. Der mechanische Abrieb von Zähnen durch direkten Zahn-zu-Zahn-Kontakt wird dabei als Attrition bezeichnet, während die Abnutzung von Zahnhartsubstanz durch Fremdstoffe als Abrasion definiert ist. Da sich die Krankheitsbilder klinisch häufig überlagern und damit eine exakte Differenzierung erschweren, ist eine sorgfältige anamnestische Abklärung notwendig, um potentielle ätiologische Faktoren zu ermitteln und eine ursachenbezogene Therapie einzuleiten. Eckpfeiler der Therapie müssen präventive Maßnahmen sein um eine weitere Progression des Substanzverlustes zu verhindern und eine höhere Überlebensrate der meist notwendigen Rekonstruktionen zu gewährleisten [5]. Erst nach Stabilisierung der Situation sollten komplexe restaurative Vorgehensweisen zur Wiederherstellung der vertikalen Dimension und der Zahnmorphologie in Betracht 
gezogen werden [7].

\section{Restaurative Therapie}

Die Rekonstruktion der vertikalen Kieferrelation erfolgt traditionellerweise durch Überkronung der betroffenen Zähne unter oft massiver Opferung von noch verbliebener gesunder Zahnhartsubstanz [6, 11]. Dank Fortschritten in der adhäsiven Zahnmedizin ist es möglich, weniger invasive Restaurationsformen wie okklusale vollkeramische Overlays im Seitenzahnbereich einzusetzen [3]. Dennoch handelt es sich auch bei diesen Rekonstruktionen um indirekt hergestellte Werkstücke, die eine Präparation der Zähne erfordern und hohe Behandlungskosten verursachen. Insbesondere für Patienten, bei denen nicht mit einer Übernahme der Kosten durch die Krankenversicherer gerechnet werden kann, wäre eine günstigere Alternative wünschenswert.

Kompositmaterialien ermöglichen eine defektorientierte, non-invasive Restauration der verloren gegangenen Zahnanteile ohne Schwächung der verbliebenen Zahnhartsubstanz. Lange Zeit blieb der Einsatz von Kompositen aufgrund ihrer eingeschränkten Verschleißfestigkeit und Randständigkeit auf die Versorgung von Frontzahndefekten sowie kleiner okklusaler Kavitäten beschränkt. Durch kontinuierliche werkstoffkundliche und verarbeitungstechnische Verbesserungen ist es $\mathrm{zu}$ einem Paradigmenwechsel gekommen, so dass Komposite inzwischen auch zur Restauration komplexer Kavitäten der Klassen I und II im okklusionstragenden Seitenzahnbereich routinemäßig verwendet werden. Klinische Langzeitstudien belegen die sichere Anwendbarkeit von direkten Kompositrestaurationen im Seitenzahngebiet bei einer jährlichen Verlustrate von $0-9 \%$, die im Bereich derjenigen von keramischen Werkstücken (0 - 7,5\%) liegt [4]. 
Obwohl die Indikation für direkte Kompositrestaurationen zunehmend erweitert wird und keine exakte Grenze mehr zu indirekten Versorgungen erkennen lässt, ist der Einsatz von Komposit zur direkten Bisshöhenkorrektur bisher kaum verbreitet. Neben der ungeklärten Langzeitprognose aufgrund fehlender Studien ist dies vor allem darauf zurückzuführen, dass die Verarbeitung dentaler Füllungskomposite ausgesprochen anspruchsvoll, techniksensitiv und zeitaufwendig ist. Mit einer speziellen Schienentechnik ist es möglich die verloren gegangene Zahnhartsubstanz einfach und effizient durch direkte okklusale Kompositaufbauten zu ersetzen. In einer Fall-Kontrollstudie mit einer durchschnittlichen Beobachtungszeit von 5,5 Jahren wurde gezeigt, dass mit dieser Methode der Bisshöhenrekonstruktion eine hohe Patientenakzeptanz und gute klinische Ergebnisse erzielt werden können [1]. Eine Befragung ergab zudem, dass Zahnärzte, die diese Restaurationstechnik in ihrer Praxis angewendet haben, mit dem Behandlungskonzept und dem klinischen Verhalten der direkten Kompositaufbauten zufrieden sind und diese Therapieform ihren Patienten auch weiterhin anbieten möchten [10]. Somit scheint die im Folgenden detailliert beschriebene Technik der direkten Bisshöhenrekonstruktion mit Komposit auch für die Anwendung in der Privatpraxis von Interesse zu sein.

\section{Behandlungsprinzip}

Nach eingehender Befundaufnahme werden Alginatabformungen von Ober- und Unterkiefer genommen und eine Gesichtsbogenübertragung sowie Kieferrelationsbestimmung durchgeführt. Nach sorgfältiger Analyse der einartikulierten Modelle wird im zahntechnischen Labor die gewünschte Bisshebung im Artikulator fixiert und ein Wax-up der ausgewählten Seitenzahnbereiche hergestellt. Bei der Anfertigung des Wax-ups werden jeweils die Frontzähne und die 
am weitesten distal gelegenen Seitenzähne (oder Höcker) jedes Quadranten nicht aufgebaut. Es ist auch möglich, diese Zähne in das Wax-up einzubeziehen, falls eine ausreichende Abstützung der Schiene durch bukkale, labiale oder orale Zahnflächen gewährleistet werden kann. Das Wax-up-Modell wird dupliziert und im Anschluss wird eine Tiefziehschiene auf dem neu angefertigten Gipsmodell hergestellt. Je nach Fall und geplanter Bisshebung kann es sinnvoll sein, vor dem klinischen Aufbau der Okklusion eine Stabilisierungsschiene anzufertigen, um den Patienten an die neue Situation zu gewöhnen und einer Kiefergelenkssymptomatik vorzubeugen [2, 8].

Bei der nachfolgenden klinischen Umsetzung der schienenunterstützten Zahnaufbauten ist folgendes Vorgehen sinnvoll: Nach Lokalanästhesie (nicht zwingend erforderlich, situationsabhängig) werden die Zähne des gesamten Zahnbogens mittels Kofferdam trockengelegt und anschließend gereinigt. Es folgt die Einprobe der Tiefziehschiene am Patienten. Ein wesentlicher Schritt ist hierbei das Prüfen der Passgenauigkeit und Sitzstabilität der Schiene. Die Schiene sollte sich spannungsfrei in Endposition bringen lassen und die verloren gegangene Zahnhartsubstanz als Hohlraum gemäß Wax-up im okklusalen Bereich zwischen Schienenkunststoff und Restzahnsubstanz repräsentieren. Gegebenenfalls ist es erforderlich, die Schiene im Kofferdamklammerbereich leicht zu modifizieren um eine verbesserte Passung zu erreichen.

Zur Vermeidung eines interdentalen Verblockens beim Aufbau der fehlenden Zahnanteile mit Komposit wird empfohlen die Nachbarzähne der zuerst zu restaurierenden Zähne mit Teflonband zu isolieren. Vorteilhaft ist neben der äußerst geringen Schichtstärke des Bandes seine ausgesprochen gute Adaptationsfähigkeit. Das Einbringen des Isolierbandes in den Approximalraum kann insbesondere beim Vorliegen straffer Kontakte durch einen interdental hebelnd angesetzten Spatel 
erleichtert werden. Alternativ können zur Separation der Zähne bzw. zur Gestaltung der Approximalflächen auch Kunststoff- oder Stahlmatrizen verwendet werden.

Es folgt die Vorbereitung der ersten Prämolaren und der ersten Molaren zur adhäsiven Befestigung. Die diesbezügliche Vorgehensweise variiert je nach verwendetem Adhäsiv- und Kompositsystem. Da erodierte Dentinoberflächen teilweise sklerotische Veränderungen aufweisen, empfehlen die Autoren zunächst ein mechanisches Anfrischen des Dentins mit rotierenden Instrumenten. Anschließend sollte der Zahnschmelz für mindestens $30 \mathrm{~s}$ mit Phosphorsäure geätzt werden. Es folgen die weiteren systemabhängigen Konditionierungsschritte und die Applikation des Adhäsivs. Bereits bestehende, klinisch und radiologisch suffiziente Kompositfüllungen sollten vor dem Auftragen des Haftvermittlers mit einem Sandstrahlgerät $\left(\mathrm{Al}_{2} \mathrm{O}_{3}-\mathrm{Pulver}\right)$ angeraut und silanisiert werden. Das Komposit wird danach in einer der fehlenden Zahnsubstanz entsprechenden Menge auf die Zähne sowie in die Schiene appliziert, die daraufhin mit gleichmäßigem Druck langsam positioniert wird. Grobe Überschüsse sollten bereits vor der Lichthärtung entfernt werden. Die Lichtpolymerisation durch die Schiene erfolgt zunächst nur für 3-4 s, so dass ein oberflächliches „Einfrieren“ des Kompositmaterials erreicht wird und die Schiene vorsichtig abgenommen werden kann. Noch vorhandene Überschüsse lassen sich danach mit einem Skalpell leicht entfernen. Dann erst erfolgt die vollständige Polymerisation des Kompositmaterials durch Bestrahlung von okklusal, bukkal und oral für jeweils weitere $60 \mathrm{~s}$. Nach Ausarbeitung (vor allem interdental) und Teflonbandisolierung der bereits rekonstruierten Zähne werden in einem weiteren Schritt die zweiten Prämolaren wie oben beschrieben konditioniert und versorgt. Zuletzt werden die zweiten Molaren direkt oder ebenfalls mittels Schiene aufgebaut. 
Falls sowohl für den Ober- als auch für den Unterkiefer eine Erhöhung der vertikalen Dimension geplant wurde, werden zunächst die Seitenzahnbereiche des einen Kiefers aufgebaut und im Gegenkiefer bestehende Füllungen oder eine bestehende Schiene eingeschliffen. Dann erst wird der Gegenkiefer versorgt um die definitive Okklusion einzustellen. Die Frontzähne können in weiteren Sitzungen direkt aufgebaut werden um die Abstützung im Frontzahnbereich sicherzustellen.

Das vorgestellte Behandlungskonzept stellt eine im Vergleich zu herkömmlichen indirekten Verfahren kostengünstigere, jedoch für den Behandler aufwendige restaurative Maßnahme dar. Abb. 1 zeigt das Vorgehen Schritt für Schritt am Modell.

\section{Klinischer Fall (Abb. 2)}

Die Patientin war zum Zeitpunkt der Befundaufnahme 63 Jahre alt und allgemeinmedizinisch gesund. Dental wurden generalisierte okklusale Erosionen mit freiliegendem Dentin vor allem im Oberkiefer sowie Primärkaries und insuffiziente Kompositfüllungen im Front- und Seitenzahngebiet diagnostiziert. Aufgrund des erkennbaren Zahnhartsubstanzverlustes war von einer Reduktion der vertikalen Dimension um ca. $3 \mathrm{~mm}$ auszugehen. Anamnestisch gab die Patientin an in Stresssituationen an Rumination (Wiederkäuen) zu leiden. Speicheltests ergaben keine pathologischen Befunde.

Die erforderliche restaurative Therapie sollte in Absprache mit der Patientin minimalinvasiven Behandlungsgrundsätzen folgen und sah eine Anhebung der Bisslage um $3 \mathrm{~mm}$ vor. Nach Austausch der insuffizienten Füllungen und Kariestherapie an Zahn 22 wurde eine Stabilisierungsschiene angefertigt, die die Patientin zur Adaptation an die neue Bisslage vier Monate lang trug. Danach wurden 
die Oberkieferseitenzähne mit einer Tiefziehschiene in der oben beschriebenen Technik in einer Sitzung direkt mit Komposit aufgebaut und der kariöse Zahn 17 wurde extrahiert. Die Oberkieferfront wurde ebenfalls in direkter Technik mit Kompositrestaurationen versorgt. Das erzielte Behandlungsergebnis stellte die Patientin sowohl in funktioneller als auch in ästhetischer Hinsicht voll zufrieden.

Fazit für die Praxis

Direkte Kompositrestaurationen kommen zunehmend in Situationen zur Anwendung, die traditionell indirekten Restaurationsformen vorbehalten waren. Die Vorteile eines direkten Therapieansatzes mit Komposit sind neben der fehlenden Invasivität und guten Reparaturmöglichkeit die für den Patienten verhältnismäßig günstigen Behandlungskosten. Die im Beitrag präsentierte Methode der Bisshebung mit direkten adhäsiven Kompositaufbauten stellt eine zumindest „semi-permanente“ Behandlungsoption zur Versorgung fortgeschrittener Erosions-/Abrasionsgebisse dar. Dadurch lassen sich Zahnhartsubstanz opfernde, invasive Maßnahmen vermeiden oder auf einen späteren Zeitpunkt verschieben. Obwohl bislang keine Daten zum klinischen Langzeitverhalten vorliegen, scheint das günstige Kosten-Nutzen-Verhältnis den Einsatz dieser Therapiemöglichkeit auch in der Privatpraxis zu rechtfertigen.

Interessenkonflikt. Der korrespondierende Autor gibt an, dass kein Interessenkonflikt besteht. 


\section{Literatur}

1. Attin T, Filli T, Imfeld C et al. (2011) Clinical behaviour of resin composite posterior vertical bite reconstructions after 5.5 years - a case series. J Oral Rehabil [Epub ahead of print]

2. Gavish A, Winocur E, Ventura YS et al. (2002) Effect of stabilization splint therapy on pain during chewing in patients suffering from myofascial pain. J Oral Rehabil 29: 1181-1186

3. Hastings $\mathrm{JH}$ (1996) Conservative restoration of function and aesthetics in a bulimic patient: a case report. Pract Periodontics Aesthet Dent 8: 729-36; quiz 738

4. Hickel R, Manhart J (2001) Longevity of restorations in posterior teeth and reasons for failure. J Adhes Dent 3: 45-64

5. Johansson A, Johansson AK, Omar R et al. (2008) Rehabilitation of the worn dentition. J Oral Rehabil 35: 548-566

6. Kavoura V, Kourtis SG, Zoidis P et al. (2005) Full-mouth rehabilitation of a patient with bulimia nervosa. A case report. Quintessence Int 36: 501-510

7. Little JW (2002) Eating disorders: dental implications. Oral Surg Oral Med Oral Pathol Oral Radiol Endod 93: 138-143

8. Miller VJ (1992) Treatment dentures: acrylic partial denture and stabilization splint. J Prosthet Dent 67: 736-737

9. Schmidlin PR, Schicht OO, Attin T (2009) Die direkte schienenunterstützte Bisshöhenrekonstruktion - eine minimalinvasive Restaurationstechnik mit Komposit. Quintessenz 60: 909-919

10. Tauböck TT, Attin T, Schmidlin PR (2011) Implementation and experience of a new method for posterior vertical bite reconstruction using direct resin 
composite restorations in the private practice - a survey. Acta Odontol Scand [Epub ahead of print]

11. Van Roekel NB (2003) Gastroesophageal reflux disease, tooth erosion, and prosthodontic rehabilitation: a clinical report. J Prosthodont 12: 255-259 


\section{Abbildungslegenden}

Abb. 1: Technik der direkten schienenunterstützten Bisshöhenrekonstruktion mit Komposit am Modell (modifiziert nach [9]). Ausgangssituation mit okklusal ungleichmäßig ausgeprägten simulierten Erosionsdefekten der Seitenzähne (a). Eingepasste Tiefziehschiene nach Kofferdamapplikation (b). Teflonbandisolierung der Nachbarzähne der zuerst zu rekonstruierenden Zähne (c). Mit Komposit befüllte und reponierte Schiene. Grobe Überschussentfernung vor der kurzen initialen Lichthärtung (d). Zustand nach Grobausarbeitung der vollständig polymerisierten Kompositaufbauten (e). Isolation der zuvor rekonstruierten Zähne mit Teflonband. Kompositapplikation auf den aktuell zu versorgenden Zahn und in die Tiefziehschiene (f). Zustand nach Grobausarbeitung aller aufgebauten Zähne (g). Fertig ausgearbeitete und polierte Kompositversorgungen nach Abnahme des Kofferdams (h).

Abb. 2: Patientenfall. Okklusalansicht des Oberkiefers vor Behandlung. Starke generalisierte Erosionen mit Dentinbeteiligung, Primärkaries (Zahn 22 und 17), insuffiziente Kompositfüllungen (Zahn 16, 15 und 21) (a). Okklusalansicht des Oberkiefers nach direkter Bisshöhenrekonstruktion mit Komposit. Vor der Bisshebung wurden die insuffizienten Füllungen ausgetauscht und die kariöse Läsion an Zahn 22 mit Komposit versorgt. Der kariöse Zahn 17 wurde extrahiert (b). 


\section{Fragen zur Lernerfolgskontrolle}

1. Was erklären Sie Ihrem Patienten über die bei ihm vorliegenden nichtkariösen Zahnhartsubstanzdefekte?

(A) Erosionen werden durch bakteriell gebildete Säuren verursacht.

(B) Häufiger Konsum von säurehaltigen Nahrungsmitteln und Getränken kann Erosionen verursachen.

(C) Abrasion entsteht durch direkten Kontakt antagonistischer oder benachbarter Zahnflächen.

(D) Attrition wird durch Fremdkörperabrieb verursacht.

(E) Erosive und abrasive bzw. attritive Prozesse treten klinisch selten kombiniert auf.

2. Wie gehen Sie bei der Therapie von nichtkariösen Zahnhartsubstanzverlusten vor?

(A) Sie klären vor jeder restaurativen Therapie die Ursachen der Zahnhartsubstanzverluste ab.

(B) Sie leiten unverzüglich nach der Diagnosestellung die symptomatische Therapie ein.

(C) Sie erklären Ihrem Patienten, dass Kompositrestaurationen nur zur Therapie von Zahnhals- und Frontzahndefekten geeignet sind.

(D) Sie sagen Ihrem Patienten, dass seine okklusal lokalisierten Defekte mit Dentinbeteiligung mit Kronen versorgt werden müssen.

(E) Sie raten Ihrem Patienten bei einem Verlust der vertikalen Bisshöhe von vollkeramischen Overlays prinzipiell ab. 
3. Welche Aussage zu direkten Kompositrestaurationen trifft zu?
(A) Sie erfordern die Präparation von Unterschnitten.
(B) Sie weisen eine gute Reparaturmöglichkeit auf.
(C) Sie sind im Vergleich zu indirekt hergestellten Werkstücken deutlich teurer.
(D) Die jährliche Verlustrate liegt deutlich über derjenigen von keramischen Werkstücken.

(E) Sie werden zunehmend durch indirekte Restaurationen verdrängt.

4. Wie hoch ist die jährliche Verlustrate von direkten Kompositrestaurationen im Seitenzahnbereich?
(A) $\quad 0-9 \%$
(B) $7-13 \%$
(C) $\quad 10-12 \%$
(D) $\quad 15-18 \%$
(E) $\quad 13-17 \%$

5. Welche Aussage zur direkten Bisshöhenrekonstruktion mit Komposit trifft nicht zu?
(A) Es besteht eine hohe Patientenakzeptanz für dieses Behandlungskonzept.
(B) Das Behandlungskonzept erreicht eine hohe Akzeptanz bei Zahnärzten, die diese Methode der Bisshöhenrekonstruktion bereits durchgeführt haben.
(C) Es liegen noch keine Daten zum klinischen Langzeitverhalten der Restaurationen vor.
(D) Spätestens nach 24 Monaten müssen die direkten Kompositaufbauten durch Kronen ersetzt werden.


(E) Das Kosten-Nutzen-Verhältnis ist bei dieser Methode der Bisshöhenrekonstruktion günstig.

6. Sie möchten bei einem Ihrer Patienten eine direkte Bisshöhenrekonstruktion mit Komposit durchführen. Welchen der folgenden Punkte zur klinischen Durchführung müssen Sie beachten?

(A) Auf eine Gesichtsbogenübertragung und Kieferrelationsbestimmung kann verzichtet werden.

(B) Vor dem Aufbau der Okklusion muss der Patient in jedem Fall für mindestens sechs Monate eine Stabilisierungsschiene tragen um einer Kiefergelenkssymptomatik vorzubeugen.

(C) Auf Kofferdamapplikation kann in der Regel verzichtet werden.

(D) Freiliegende erodierte Dentinoberflächen sollten vor der Konditionierung mechanisch angefrischt werden.

(E) Bestehende klinisch und radiologisch suffiziente Füllungen sollten vor der Bisshebung ausgetauscht werden.

7. Wie lange sollte der Schmelz mit Phosphorsäure angeätzt werden?

(A) Der Schmelz sollte für höchstens $5 \mathrm{~s}$ angeätzt werden.

(B) Der Schmelz sollte für höchstens $10 \mathrm{~s}$ angeätzt.werden.

(C) Der Schmelz sollte für höchstens $20 \mathrm{~s}$ angeätzt werden.

(D) Der Schmelz sollte für mindestens $30 \mathrm{~s}$ angeätzt werden.

(E) Der Schmelz sollte für mindestens 3 min angeätzt werden.

8. Welche Aussage zu der bei der direkten Bisshöhenrekonstruktion mit Komposit verwendeten Tiefziehschiene trifft zu? 


\section{(A) Sie basiert auf einem individuellen Wax-up und dient als Formhilfe für die direkten Kompositaufbauten.}

(B) Sie dient dem Schutz der direkten Kompositaufbauten und sollte nach der Bisshöhenrekonstruktion 6 Monate nachts getragen werden.

(C) Sie muss vor dem Aufbau der Okklusion für mindestens sechs Monate als Stabilisierungsschiene getragen werden.

(D) Eine Abstützung der Schiene im Mund des Patienten ist nicht erforderlich.

(E) Sie wird am Phantommodell hergestellt.

9. Wozu dient das bei der direkten Bisshöhenrekonstruktion mit Komposit verwendete Teflonband?
(A) Es dichtet Risse im Kofferdam ab.
(B) Es verhindert ein interdentales Verblocken beim Aufbau der Zähne.
(C) Es dient zum Überprüfen der Approximalkontakte.
(D) Es dient zum Überprüfen der statischen und dynamischen Okklusion.
(E) Es dient dem Schutz der direkten Kompositaufbauten vor Überbelastung.

10. Was sollten Sie bei der Polymerisation der direkten Kompositaufbauten beachten?

(A) Die Lichtpolymerisation sollte 3-4 s nicht überschreiten.

(B) Nach einer kurzen initialen Lichtpolymerisation von 3-4 s lassen sich vorhandene Überschüsse leicht mit einem Skalpell entfernen.

(C) Die Lichtpolymerisation sollte nur bei aufgesetzter Tiefziehschiene erfolgen um den Polymerisationsgrad des Kompositmaterials zu erhöhen.

(D) Zur Minimierung der Schrumpfung des Kompositmaterials sollte die Lichtpolymerisation ausschließlich von bukkal erfolgen. 
(E) Es sollten ausschließlich selbsthärtende Komposite verwendet werden. 



Abb. 1 

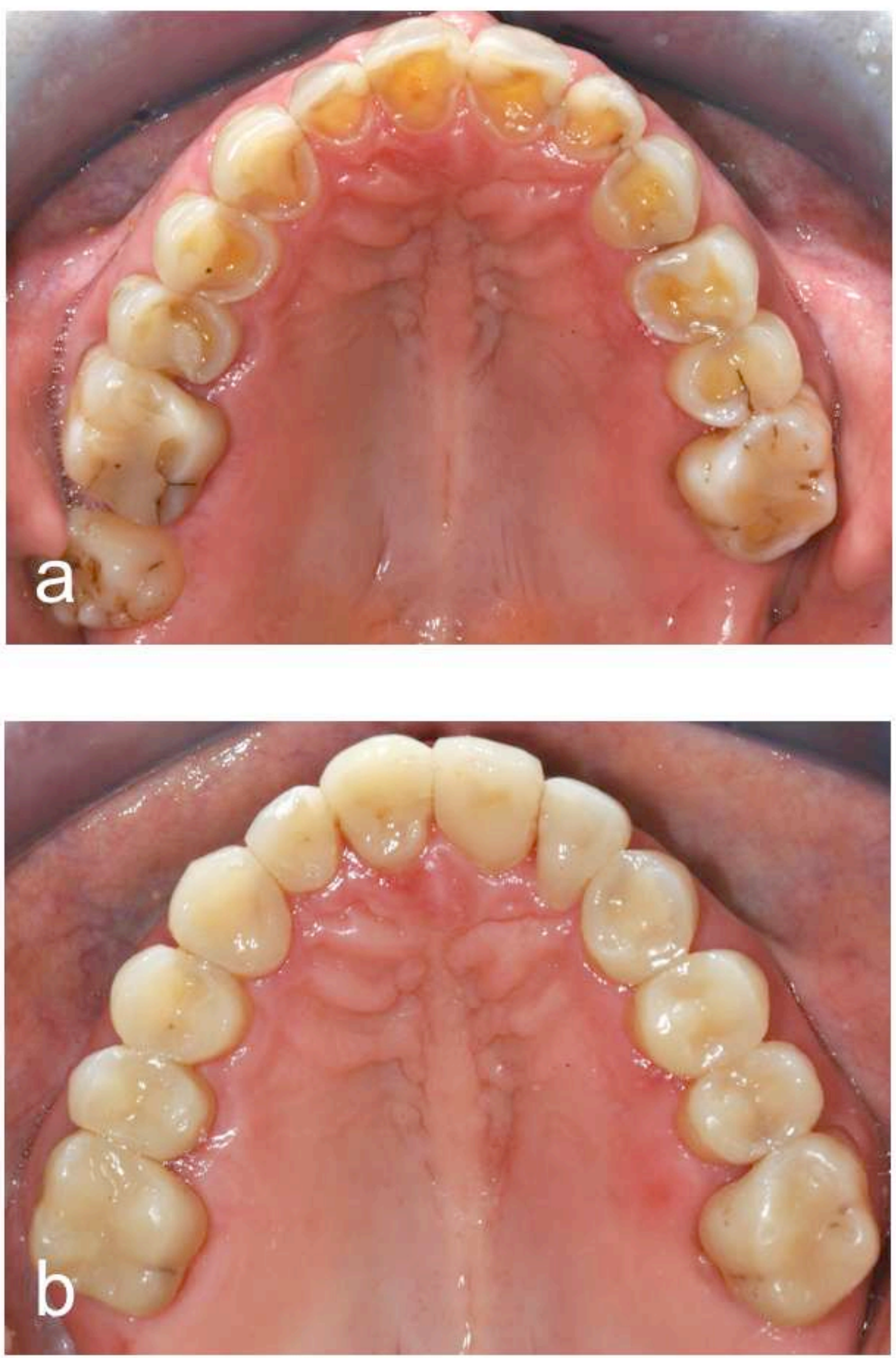

Abb. 2 